\title{
Observation of Radial Ring Deformations using Closed Orbits at LEP
}

\author{
J. Wenninger*, CERN, Geneva, Switzerland
}

\begin{abstract}
With a circumference of $26.7 \mathrm{~km}$ the LEP ring is subject to long range geological deformations which must be monitored accurately to guarantee a sufficient precision on the beam energy for the LEP experiments. Changes of the LEP circumference can be extracted from the radial position of the closed orbits with an accuracy better than $0.1 \mathrm{~mm}$. The observed tidal deformations with amplitudes of up to $1 \mathrm{~mm}$ were found to be in good agreement with geological models. Besides such periodic effects seasonal circumference variations of $2 \mathrm{~mm}$ are also observed. A summary of measurements collected between 1993 and 1998 is presented.
\end{abstract}

\section{INTRODUCTION}

The influence of terrestrial tides on the LEP beam energy was discovered in 1991 when calibrations were performed for the first time by resonant depolarization [1, 2]. In 1992 and 1993 further studies demonstrated that the beam energy variations were in good agreement with the predictions of tide models [3]. But it also became clear that in addition to tidal deformations the ring was subject to long term circumference oscillations with an annual periodicity. Energy calibrations by resonant depolarization have an accuracy of $10 \mathrm{ppm}$ but can only be performed when the beam are not colliding. For very precise measurements of the $\mathrm{Z}$ boson mass and width this calibration must be extrapolated in time to the periods where the beams collide and the LEP experiments take data without spoiling the excellent accuracy [4]. Information extracted from closed orbits has been used to track and understand circumference changes which alter the LEP beam energy $[5,6]$. This paper reviews the present knowledge on radial deformation at LEP.

\section{RADIAL DEFORMATIONS}

Since in LEP the particles are ultra-relativistic, their orbit length is determined by the frequency of the RF system which is normally kept constant. As a consequence of a circumference change $\Delta \mathrm{C}$, the beam is forced to adapt its radial position in the quadrupoles. The additional bending field leads to a change in energy $\Delta \mathrm{E}$ :

$$
\frac{\Delta \mathrm{E}}{\mathrm{E}}=-\frac{1}{\alpha} \frac{\Delta \mathrm{C}}{\mathrm{C}}=-\frac{\epsilon}{\alpha}
$$

\footnotetext{
*Email: Jorg.Wenninger@cern.ch
}

where $\alpha$ is the momentum compaction factor. $\epsilon$ is the horizontal strain. For the LEP optics used between 1993 and $1998 \alpha$ varied between $1.43 \cdot 10^{-4}$ and $1.86 \cdot 10^{-4}$.

The horizontal movement of the beam relative to the quadrupoles can be observed either through the energy change or directly with the beam orbit measurement system. To enhance the sensitivity and reduce the systematic errors, the horizontal beam position is averaged over all BPMs (Beam Position Monitors) located in the regular arc FODO cells at the vertically focussing quadrupoles $[5,6]$. Up to 240 BPMs can be used to compute this average beam position which will be denoted $\mathrm{X}_{\mathrm{ARC}}$. The horizontal dispersion $\mathrm{D}_{\mathrm{x}}$ is ideally identical at all the selected BPMs. $\mathrm{X}_{\mathrm{ARC}}$ is related to $\Delta \mathrm{C}$ by

$$
\Delta \mathrm{X}_{\mathrm{ARC}}=\mathrm{D}_{\mathrm{x}} \frac{\Delta \mathrm{E}}{\mathrm{E}}=-\frac{\mathrm{D}_{\mathrm{x}}}{\alpha} \frac{\Delta \mathrm{C}}{\mathrm{C}}
$$

The factor $\mathrm{D}_{\mathrm{x}} / \alpha$ can be measured using a controlled change of the RF frequency $f_{R F}$ since this leads to a position shift of

$$
\Delta \mathrm{X}_{\mathrm{ARC}}=-\frac{\mathrm{D}_{\mathrm{x}}}{\alpha} \frac{\Delta \mathrm{f}_{\mathrm{RF}}}{\mathrm{f}_{\mathrm{RF}}}
$$

The ratio $\mathrm{D}_{\mathrm{x}} / \alpha \approx 2.5-2.9 \cdot 10^{3}(\mathrm{~m})$ depends slightly on the beam optics. The measurements agree with MAD [7] calculations within $2 \%$.

\section{EARTH TIDES}

Tides are caused by the $1 / r^{2}$ dependence of the gravitational attraction of the sun and the moon. The presence of a celestial body leads to a quadrupolar deformation of the Earth surface. The radius change $\Delta R$ is

$$
\Delta \mathrm{R} \sim \frac{\mathrm{M}}{\mathrm{a}^{3}} P_{2}(\cos \theta)=\frac{\mathrm{M}}{2 \mathrm{a}^{3}}\left(3 \cos ^{2} \theta-1\right)
$$

where $\theta$ is the angle between the vertical and the celestial body and a is the distance to the body of mass M. $P_{2}$ is the $2^{\text {nd }}$ order Legendre polynomial. Figure 1 shows a sketch of the lunar tide bulge. Sun tides are about $50 \%$ weaker than Moon tides. Under the influence of the tidal forces the earth surface moves vertically by about $\pm 12 \mathrm{~cm}$ in the area around Geneva. The associated horizontal strain $\epsilon$ induces peak-to-peak circumference changes of up to $1 \mathrm{~mm}$.

The influence of the tides on LEP can be described by a CTE (Cartwright-Taylor-Edden) model which includes the 505 main tide harmonics [8]. A program based on such a 


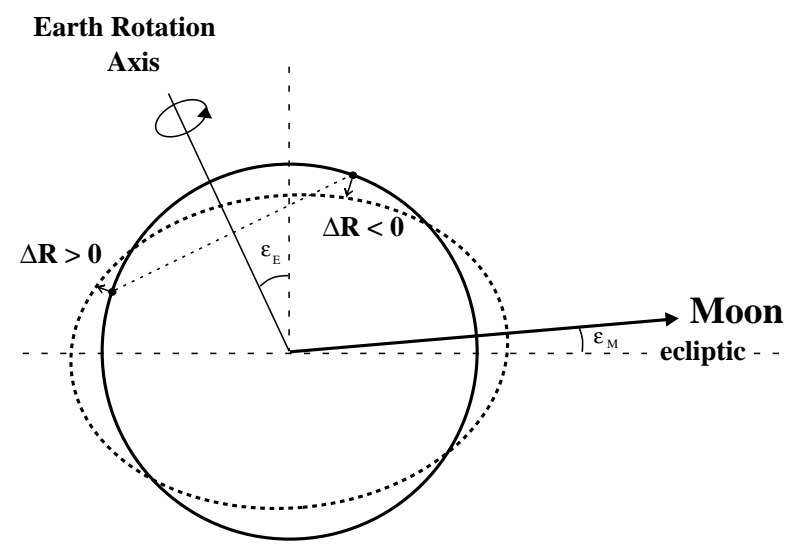

Figure 1: Tidal deformation of the Earth surface due to the presence of the Moon. The inclination $\varepsilon_{E}$ of the Earth rotational axis with respect to the plane of the ecliptic is $23.5^{\circ}$. The inclination $\varepsilon_{M}$ of the lunar orbital plane is $5.1^{\circ}$.

tide model, where some parameters are adjusted with actual gravity measurements, is available to estimate the tidal change in gravitational acceleration $\Delta \mathrm{g}$ which must then be related to the strain $\epsilon$. Figure 2 shows an example of tide predictions for LEP. At high tides $|\Delta g|$ reaches 1.5 $\mu \mathrm{m} / \mathrm{s}^{2}$ around LEP for an average acceleration of gravity $\mathrm{g}=9.805 \mathrm{~m} / \mathrm{s}^{2}$.

The fraction of gravity change that couples into a horizontal strain $\epsilon$ can be described by a coupling parameter $\lambda_{\mathrm{T}}$ :

$$
\epsilon=\frac{\Delta \mathrm{C}}{\mathrm{C}}=\lambda_{\mathrm{T}} \frac{\Delta \mathrm{g}}{\mathrm{g}}
$$

where $\lambda_{\mathrm{T}} \approx-0.16$ from geological observations [8]. The maximum strain $\epsilon$ reaches a tiny $\approx \pm 2.2 \cdot 10^{-8}$.

The beam position measurement $\mathrm{X}_{\mathrm{ARC}}$ can now be re-

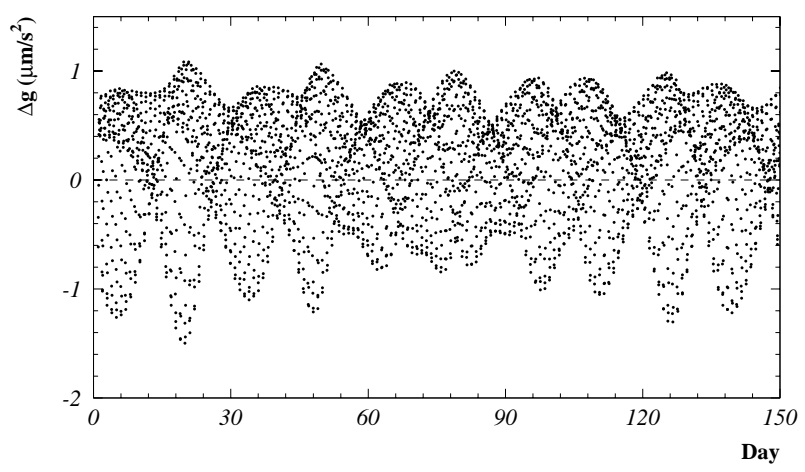

Figure 2: Local gravity change $\Delta \mathrm{g}$ predicted for LEP. The time scale starts on January $1^{s t} 1996$. The largest amplitudes occur every 14 days at full and new Moon. The first high tide on January $5^{\text {th }}$ corresponds to full Moon. The amplitudes are modulated by the eccentricity of the lunar orbit and the seasonal variation of the Sun position.

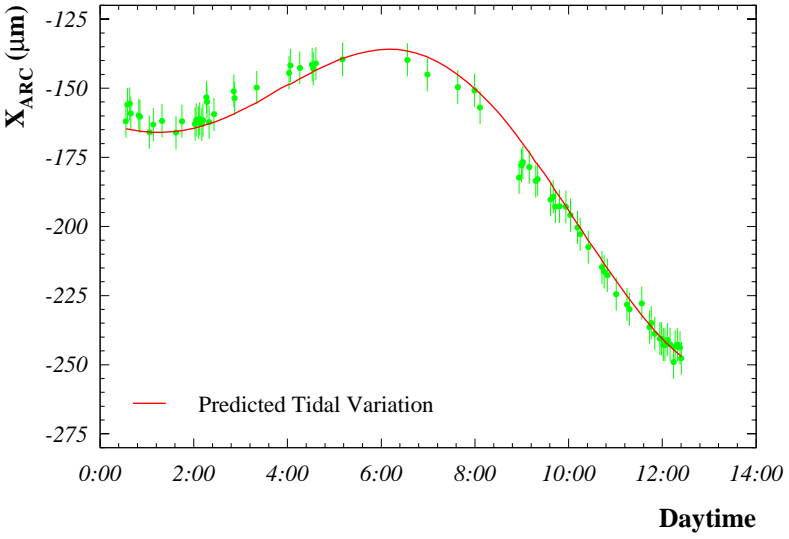

Figure 3: Example of the evolution of $X_{A R C}$ during a LEP fill. The data points follow the variation expected from the CTE tide model.

lated to $\Delta \mathrm{g}$ :

$$
\Delta X_{A R C}=-\frac{\mathrm{D}_{\mathrm{x}}}{\alpha} \frac{\Delta \mathrm{C}}{\mathrm{C}}=-\frac{\mathrm{D}_{\mathrm{x}} \lambda_{\mathrm{T}}}{\alpha} \frac{\Delta \mathrm{g}}{\mathrm{g}}
$$

Figure 3 shows an example of a tidal distortion of the ring observed with the BOM system over a LEP fill.

A sample closed orbits acquired in stable beam conditions in 1994 and 1995 has been used for the analysis of tide effects. The sample of ARC BPMs is scanned for channel which show frequent problems or a suspicious behaviour. Such BPMs (about 40 out of the 240) are completely removed from the analysis. To avoid biases due to slow changes of the circumference that are not related to tides, only the changes of $\mathrm{X}_{\mathrm{ARC}}$ with respect to its fill average (maximum 12 hours) are fitted to $\Delta \mathrm{g}$. The residuals of the individual measurements after the fit are typically 5 to $7 \mu \mathrm{m}$. Figure 4 shows the spread of $\mathrm{X}_{\mathrm{ARC}}$ around its

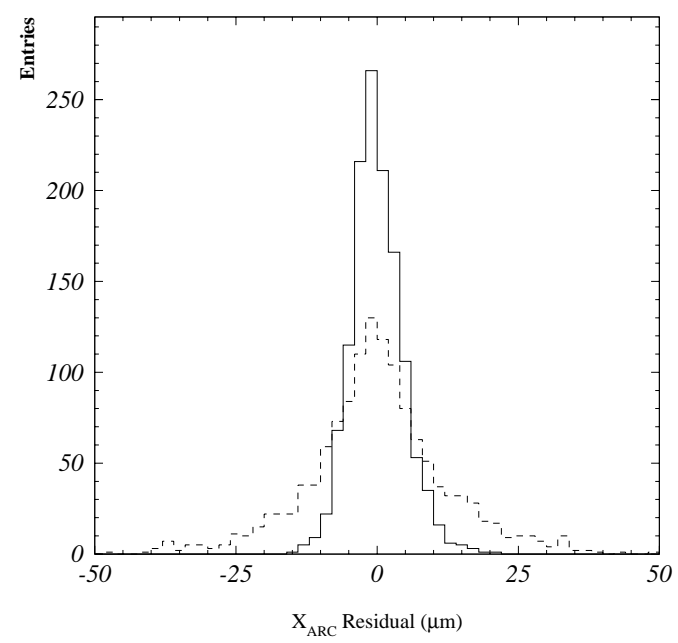

Figure 4: Distribution of the residuals of $\mathrm{X}_{\mathrm{ARC}}$ with respect to the fill average before (dashed histogram) and after tide correction (solid histogram). The RMS is reduced from 14 to $5 \mu \mathrm{m}$. 


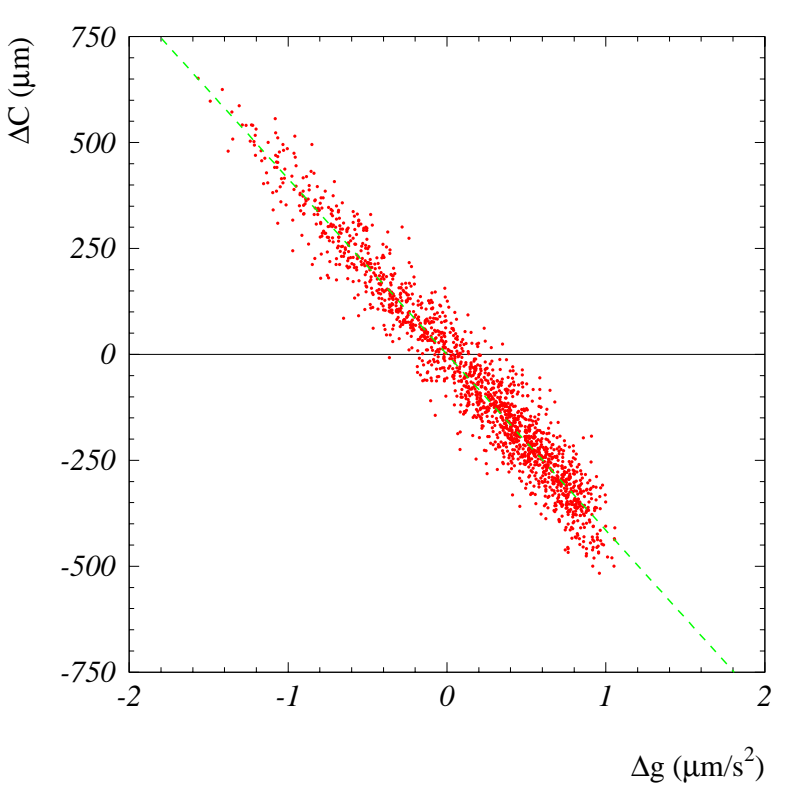

Figure 5: Correlation between the change in circumference $\triangle \mathrm{C}$ measured with the BOM system and the predicted tidal gravity correction $\Delta \mathrm{g}$. The slope is $-410 \pm 20 \mathrm{~s}^{2}$.

average value over a fill before and after correction for the tides.

The correlation between $\Delta \mathrm{g}$ and $\Delta \mathrm{C}$ is shown in Figure 5. The slope $\lambda_{\mathrm{T}} \mathrm{C} / \mathrm{g}$ (Equation 6) is measured to be $-410 \pm 20 \mathrm{~s}^{2}$, yielding $\lambda_{\mathrm{T}}=-0.15 \pm 0.01$, in good agreement with independent geological measurements. The error is dominated by systematic effects due to tails in the distribution of the fit residuals (Figure 4).

The measurements obtained from this analysis agree well with the results from energy calibration by resonant depolarization $[3,5]$.

\section{LONG TERM RING DEFORMATION}

Besides the periodic tidal movements LEP is also subject to much slower long term circumference changes. Figure 6 displays the circumference changes $\triangle \mathrm{C}$ of LEP between 1993 and 1998. $\Delta \mathrm{C}$ has been calculated from $\mathrm{X}_{\mathrm{ARC}}$ after correction for tidal effects. Data from different runs or years can be related by measuring the RF frequency for which the beam is on average centred in the quadrupoles. Between May and November the LEP ring experiences circumference changes of up to $2 \mathrm{~mm}$, the general trend being reproducible from one year to the next. The circumference usually increases during the Summer months. Some of the changes are clearly correlated to rainfall and to fluctuations in the underground water table height. It was not possible to develop a model able to predict $\Delta \mathrm{C}$ with good accuracy because the geological problem is complex and too many variables are unknown.

Those seasonal variations of the circumference are very important to understand the LEP beam energy. The circumference changes shown in Figure 6 are an important

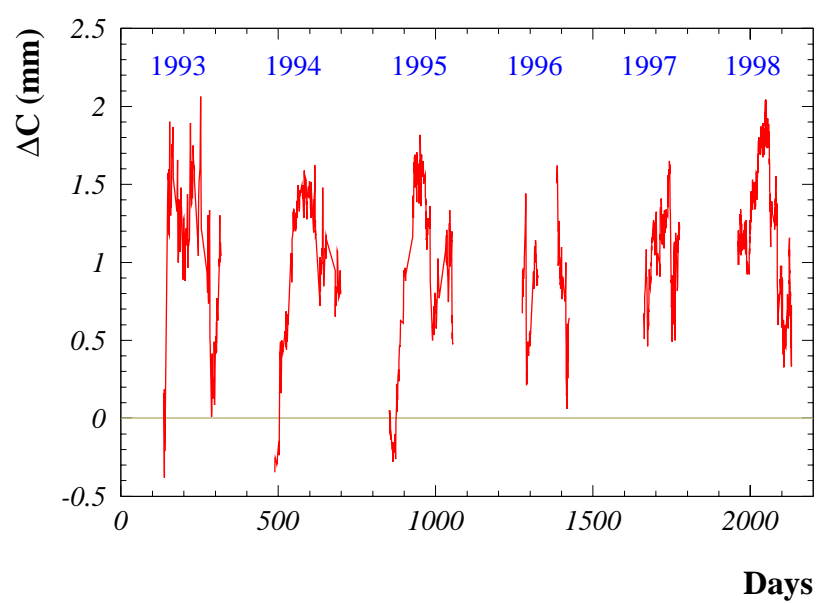

Figure 6: Relative change of the LEP circumference $\Delta \mathrm{C}$ between 1993 and 1998 .

ingredient to model the LEP beam energy. The associated energy variation is $\Delta \mathrm{E} / \mathrm{E} \approx 4-5 \cdot 10^{-4}$, which is more than one order of magnitude larger than the desired accuracy on $\mathrm{E}$.

\section{CONCLUSION}

Closed orbits have been used for a quantitative determination of the deformation of LEP due to terrestrial tides. The measured decrease of the LEP circumference of $4.1 \pm$ $0.2 \mu \mathrm{m}$ for a relative increase in the local gravity of $10^{-9}$ is in good agreement with previous measurements by resonant depolarization and with geological observations.

Besides those periodic tide effects even larger seasonal circumference variations of about $2 \mathrm{~mm}$ peak-to-peak have been observed from the beam position relative to the quadrupoles.

The information gained from the closed orbit data has been successfully used to track beam energy changes and to guarantee a precise measurement of the $\mathrm{Z}$ boson mass and width.

\section{REFERENCES}

[1] L. Arnaudon et al., Phys. Lett. B 307 (1993) 187.

[2] L. Arnaudon et al., Phys. Lett. B 284 (1992) 431; L. Arnaudon et al., Z. Phys. C 66 (1995) 45.

[3] L. Arnaudon et al., Nucl. Instr. Meth. A 357 (1995) 249.

[4] R. Assmann et al., Z. Phys. C 66 (1995); R. Assmann et al., Eur. Phys. J. C 6 (1999) 2, 187.

[5] J. Wenninger, "Study of the LEP Beam Energy with Beam Orbits and Tunes", CERN SL/94-14 (BI).

[6] J. Wenninger, "Radial Deformations of the LEP Ring", CERN SL/Note 95-21 (OP).

[7] H. Grote, C. Iselin, The MAD program V8.10, CERNSL/90-13 Rev. 3 (AP).

[8] P. Melchior, The Tides of the Planet Earth, 2nd Edition, Pergamon Press (1983). 